\title{
Quelques aspects de la contradiction et de la remise en cause dans le genre Letters to the Editor
}

\section{Anne Magnet et Didier Carnet}

\section{(2) OpenEdition}

\section{Journals}

\section{Édition électronique}

URL : http://journals.openedition.org/asp/1773

DOI : $10.4000 /$ asp. 1773

ISBN : 978-2-8218-0384-8

ISSN : 2108-6354

\section{Éditeur}

Groupe d'étude et de recherche en anglais de spécialité

Édition imprimée

Date de publication : 1 octobre 2001

Pagination : 51-62

ISSN : 1246-8185

Référence électronique

Anne Magnet et Didier Carnet, «Quelques aspects de la contradiction et de la remise en cause dans le genre Letters to the Editor », ASp [En ligne], 31-33 | 2001, mis en ligne le 09 octobre 2010, consulté le 02 mai 2019. URL : http://journals.openedition.org/asp/1773 ; DOI : 10.4000/asp.1773

Ce document a été généré automatiquement le 2 mai 2019.

Tous droits réservés 


\title{
Quelques aspects de la contradiction et de la remise en cause dans le genre Letters to the Editor
}

\author{
Anne Magnet et Didier Carnet
}

\section{Introduction}

1 Le travail de recherche dans le domaine de l'analyse du discours scientifique s'est, à ce jour, concentré sur l'article de recherche, érigé en genre, à la suite des travaux de J. Swales (1990). Le temps est venu pour notre communauté de chercheurs de s'intéresser à d'autres formes du discours scientifique primaire. L'objectif de cet article est donc de rapporter les stratégies de remise en cause de l'article de recherche ainsi que l'expression linguistique de la contradiction, telles qu'elles se présentent dans un corpus d'un genre qui, à notre connaissance, n'a pas encore fait l'objet d'étude dans le domaine des langues spécialisées : celui des Letters to the Editor. Le corpus est issu de deux revues scientifique et médicale: The American Journal of Clinical Nutrition (AJCN) et The Lancet. Nous avons répertorié et analysé les moyens linguistiques mis en œuvre par les auteurs et nous faisons ressortir les stratégies discursives d'une communauté en nous appuyant sur les travaux de J. Martin (1996) et G. Myers (1989) ainsi que sur la théorie énonciative telle qu'elle peut être adaptée en linguistique appliquée à une forme spécifique du discours scientifique.

\section{La contradiction comme concept de remise en cause}

2 La notion de représentation textuelle implique l'idée de " parcours orienté proposé par le sujet dans chaque énonciation » (Vignaux $1988: 16$ ). Le parcours orienté des Letters to the Editor se construit sur la contradiction apportée au fait scientifique établi. 
Le concept de contradiction en linguistique fait référence à la sémantique et indique donc une relation de sens entre deux items lexicaux. Des termes contradictoires sont opposés par le sens : male/female, short/tall, optimistic/pessimistic, good/bad. Il est intéressant de noter qu'en chinois, l'association des deux termes opposés est nécessaire pour désigner la notion. Ainsi «grandeur» se dit "grand-petit» (Culioli 1971: 4212). Tout concept se présente alors comme un couple (grand-pas grand) qui peut être asserté soit positivement, soit négativement.

4 Nous nous intéressons ici à la contradiction discursive que nous interprétons tout d'abord dans son sens de «dire contre » du latin dicere contra. Le sens que nous donnons ici à la contradiction est issu de la philosophie et plus précisément de la logique. Le principe de non-contradiction pose que deux propositions contradictoires ne peuvent à la fois être toutes deux vraies ou fausses.

Dans la logique de Hegel (1817: 64-65), la contradiction est le moteur de la pensée et du réel, toute position de vérité n'étant qu'un moment provisoire de la possession du réel par l'esprit. Les trois phases du progrès de la marche vers la connaissance se feront selon le processus thèse, anti-thèse et synthèse. Le discours que nous nous proposons d'analyser se situe donc dans la logique de véridiction (c'est-à-dire dans la démarche de dire le vrai) mais se situe, dans ce cas particulier, dans les mouvements de remise en cause de l'équilibre précaire atteint lors de la publication de l'article visé. Ce processus, qualifié classiquement d'antithèse, constitue souvent les prémisses de la remise en cause du fait scientifique établi.

\section{L'inscription des Letters to the Editor dans la stratégie d'une communauté de spécialistes}

Cette rubrique existe dans toutes les revues scientifiques. En anglais, elle apparaît sous le terme de Letters to the editor, ce qui est le cas dans The American Journal of Clinical Nutrition ou Correspondence, pour The Lancet. Il est intéressant de noter que dans les revues françaises, cette rubrique porte de multiples appellations ce qui montre sans doute la gêne à traduire le terme "editor». Nous avons relevé à titre d'exemples « correspondance, lettres à l'éditeur, courrier des lecteurs, lettres à la revue, lettres à la rédaction, lettres, questions-réponses ».

7 Pour replacer les Letters to the Editor dans leur contexte, il faut rappeler que la revendication scientifique se fait essentiellement au moyen de l'article de recherche, par l'utilisation d'un protocole admis et validé par la communauté, par la publication des résultats expérimentaux dans des revues hiérarchisées selon leur impact et enfin, par le processus dit de reviewing. Les Letters to the Editor se situent en marge de ce système. Il s'agit comme leur nom l'indique du courrier que reçoit le rédacteur en chef d'une revue dans lequel les auteurs apportent la contradiction par rapport aux faits établis dans un article précédemment paru. La question qui se pose est de savoir pourquoi les auteurs de ces lettres choisissent ce mode d'expression. Les réponses suivantes peuvent être avancées.

C'est le moyen le plus rapide de prendre position sur un thème de recherche sur lequel ces auteurs travaillent également et à propos duquel il y a compétition pour apporter une réponse. La publication d'un article prend au minimum plusieurs mois. Pour publier un 
article, il faut posséder soi-même suffisamment de preuves et la lettre permet de prendre date avant que tous les éléments de preuve puissent former un tout cohérent.

Ce peut être également pour le chercheur le moyen de mettre ou de remettre en lumière sa propre recherche déjà publiée et qui risque de se trouver déconsidérée ou reléguée du fait de la publication d'un article qui ne produit pas des résultats qui vont dans le même sens.

Les Letters to the Editor représentent une forme de contradiction per se par rapport à l'article de recherche. En apparence, elles se situent en marge du discours validé par la communauté, mais elles peuvent être perçues comme l'un des facteurs dynamiques de production de discours parce qu'elles ne se situent pas seulement en aval du discours produit, mais bien souvent en amont: elles relancent la recherche sur le thème. Cette forme de discours représente une contradiction constructive.

La controverse repose généralement sur :

- la méthode expérimentale choisie,

- le temps de l'expérience (généralement trop court),

- le nombre de sujets expérimentaux (généralement trop peu nombreux),

- les résultats trop fragiles, trop ténus pour être exploités.

\section{La composition du corpus}

11 Notre corpus actuel se compose de 50 lettres issues de The American Journal of Clinical Nutrition (revue américaine et $1^{\text {re }}$ revue mondiale de nutrition) et de 50 lettres issues de The Lancet (revue britannique et $2^{\mathrm{e}}$ revue mondiale de médecine). Elles couvrent la période 1999-2001.

12 Pour cette étape pionnière de notre travail, le corpus a été traité manuellement. Il est actuellement en cours de numérisation, ce qui va nous permettre d'accroître sa représentativité en remontant dans le temps et en l'étendant à d'autres revues.

\section{La macrostructure des Letters to the Editor et la relation énonciateur/co-énonciateur}

\subsection{Un genre}

Cette forme peut être considérée comme un genre, même si c'est un genre mineur dans la publication scientifique. Elle est issue du genre plus large initié en 1835 dans la presse, et dit «lettre ouverte », qui se définit comme : un article de journal, rédigé en forme de lettre et généralement de caractère polémique ou revendicatif.

Dans les revues scientifiques étudiées, elles font leur apparition dans les années 1920. L'étude de leur évolution montre que progressivement cette rubrique devient une constante dans la revue et qu'elle s'allonge avec le temps. D'une part, les lettres deviennent plus longues et leur nombre s'accroît. D'autre part, il semble que le but de ces lettres ait également évolué avec le temps. De simples mises au point (set points) pour faire avancer les connaissances sur un thème de recherche, les Letters to the Editor sont progressivement devenues un mode de mise en question de la recherche publiée, trace des enjeux stratégiques au sein d'une communauté. 
15 En choisissant un corpus de lettres issues de deux revues éminentes dans leur domaine respectif, nous tentons de dégager quelques constantes, mais aussi les variables de ce mode particulier de la communication scientifique qui s'exerce à l'intérieur de la communauté. À ce titre il vise donc la validation du fait scientifique ou la remise en cause de celui-ci et non la dispersion du discours à l'extérieur de cette communauté.

Contrairement à l'article de recherche qui lorsqu'il est publié, est accepté et validé par la communauté toute entière, les Letters to the Editor, n'engagent que leur(s) auteur(s).

Elle débute par l'utilisation de la formule caractéristique de la lettre : Dear Sir pour la revue américaine et Sir pour la revue britannique, cette deuxième cherchant peut-être d'avantage à mettre l'accent sur le côté polémique et revendicateur de la lettre. Si l'on compare cette forme d'écrit à l'article scientifique de recherche, il est tout d'abord marqué par la forte prise en charge de l'énoncé par l'énonciateur.

\subsection{La présence de l'énonciateur}

Dans les Letters to the Editor, l'énonciateur prend totalement l'énoncé en charge. Le coénonciateur est en apparence le rédacteur en chef de la revue, en réalité la communauté tout entière.

Cette prise en charge de l'énoncé se marque :

- par la forte présence du pronom personnel : we, et dans une moindre mesure de I;

- par l'usage intensif du déterminant possessif our ;

- par le recours fréquent à l'opposition de we / you, ou de we / they ou he, qui introduisent la marque de la stratégie de contradiction entre le vrai tel qu'il se veut rapporté par les auteurs pris pour cible, et les auteurs de la lettre.

Ce recours très fréquent à la mise en opposition par l'utilisation du couple we/you, ou we/ they (he) révèle une stratégie qui vise à affaiblir la construction du fait scientifique par l'article publié. En effet, par ce processus, les auteurs cherchent à prouver qu'il n'y a plus un seul fait établi, mais au moins deux.

Le recours à la forme personnalisée de l'énoncé a pour conséquence directe le faible nombre de structures à la diathèse passive. Celles-ci n'apparaissent plus que dans l'évocation des expériences, rapportées au simple past, et qui se trouvent ainsi reléguées dans l'accompli, avec lequel on peut éventuellement prendre ses distances.

21 Notre étude étant prospective et appliquée à une forme de discours encore vierge de toute recherche, nous souhaitons éviter l'écueil du catalogue. Cependant nous aimerions mettre en avant les différents points qui nous ont semblé pertinents à ce stade de défrichage.

\section{Les formes verbales}

\subsection{Temps et aspect}

Le temps le plus utilisé est le présent simple, mais dans une proportion qui rappelle l'utilisation rhétorique des temps dans la section Discussion de l'article de recherche (Magnet 1993 : 91). Le pourcentage est d'environ $50 \%$, alors que l'utilisation du simple past se situe entre 35 et $45 \%$. Le simple past est choisi pour rapporter les expériences passées de l'auteur contesté ou de celui qui engage la controverse. 
Le présent simple est utilisé pour prendre en compte la réalité de l'article contesté. Ce n'est ni un présent historique, ni un présent narratif. Cette forme de présent simple renvoie au fait scientifique établi. Cette utilisation particulière du temps présent rappelle le style journalistique. L'inscription dans le temps importe peu, ce qui compte, c'est l'absence d'aspect (ou l'utilisation de l'aspect ø) et le renvoi au fait scientifique validé (en terme de stratégie) et à la notion (en terme de linguistique) avec degré minimal de détermination.

On note le très faible recours à have + en. L'accent est donc mis sur la rupture par le jeu présent - passé, ce qui ouvre la voie à la contradiction.

L'utilisation du présent est proche de celle décrite par Quirk:

The implication of the present tense seems to be that although the communication event took place in the past, its result - the information communicated-is still operative. Thus the notion that the past can remain alive in the present also explains the optional use of the present tense in sentences referring to writers and composers and their extant works. (1997: 181)

Ex. 1

Your November 18 editorial suggests reasons for the rise in... (The Lancet $2001: 556)$

In your June editorial "is blockade of pancreatic lipase the answer?", you suggest that...(AJCN $2000: 844)$

\subsection{Les auxiliaires modaux}

La modalité épistémique est assez peu représentée. Les modaux les plus utilisés sont par ordre décroissant: should, could, may, would, et d'une manière moins marquée : can, must, will, might. Ils représentent environ $10 \%$ des structures verbales dans les Letters to the Editor, c'est-à-dire un pourcentage un peu moins élevé que dans la section Discussion de l'article de recherche où le pourcentage d'auxiliaires modaux s'élève à $14 \%$ (Magnet 1993 : 45).

Ces formes ne représentent généralement pas une modalité de l'événement, mais une modalité du sujet. On ne s'intéresse pas à la relation entre l'énonciateur et la relation prédicative qu'il propose, mais à la relation entre le sujet de l'énoncé et le prédicat. Il ne s'agit donc pas d'une valeur épistémique, mais radicale qui prend le plus souvent une valeur déontique (au sens fort de conseil moral, cf. déontologie), indiquant ainsi des rapports de pression.

\section{Ex. 2.a}

Valeur radicale, faiblement déontique :

The role of these antioxidants could be explored further by looking at the response to... (The Lancet 2001 : 631)

This goal can be facilitated by reducing the amount of fat in the diet. (AJCN $2000: 572)$

Ex. 2.b

Valeur déontique :

First the degree of oxidant stress should be assessed to choose an effective antioxidant regimen (The Lancet $2001: 631$ )

Respectfully, I must suggest we should all beware of inaccurate invectives (The Lancet $2001: 647$ ) 


\section{La contradiction par la négation de la relation prédicative}

L'un des moyens les plus récurrents de contredire est la négation. En effet, la négation est l'une des deux valeurs polaires de l'assertion. Le positif est équivalent à l'affirmation et le négatif à la négation. L'assertion se distingue par une double propriété : « Toute négation peut se ramener à une affirmation : Il est vrai qu'il est faux que Jean soit parti » (Culioli 1971 : 4212). La négation peut être liée à l'indétermination, puisque de savoir ce qu'un être n'est pas, n'entraîne pas que l'on sache ce qu'il est.

Dans notre corpus, il s'agit de nier la relation prédicative et de réfuter sa portée scientifique. Cette forme constitue la manière la plus forte de contredire un collègue scientifique. Cependant, les Letters to the Editor ne représentent pas un moyen de construire un nouveau concept scientifique, car elles ne sont soumises à aucun processus attesté de validation.

La contradiction peut être totale :

Ex. 3

Willett's editorial did not address the range of experimental animal and human studies we reviewed. (AJCN 1999 : 572)

Cette contradiction totale peut cependant être modulée. Dans ce cas, la négation porte sur la modalité et non sur le procès :

Ex. 4

The method they use does not seem to be suited to the end results. (The

Lancet $2001: 553)$

La négation peut recouvrir trois opérations dont nous trouvons effectivement trace dans notre corpus :

(a) le jugement

Ex. 5

It makes no sense to me that it is possible to reliably detect the so-called independent associations of individual constituents of this group, when ... ( AJCN $2000: 849$ )

I find Boddie et al's data depicting ... to be suggestive but not convincing evidence of impaired absorption of polyglutamyl folate. (AJCN $2001: 658$ )

(b) la prédication de l'absence

Ex. 6

In contrast, Weinsier et al's subjects were not restricted with regard to physical activity. (AJCN $2001: 657$ )

(c) le rejet

Ex. 7

In summary, the concerns of Schrauen et al are not borne out by our data. (AJCN $2000: 240)$

It is not possible to show that the protein was present in the healthy human aged brain by these techniques (AJCN $2000: 202$ )

Furthermore, this report did not clarify the quality of the clinical trials included in the analysis. (AJCN $2001:$ 653) 
The lessons I derived from receiving my Ross Research Conference proceedings as an AJCN supplement were profound but not all of them were of an academic nature. (AJCN $2001: 655$ )

\section{Les marqueurs lexicaux explicites de la contradiction}

La contradiction prend également une forme explicite à travers l'utilisation renforcée de marqueurs particuliers au niveau sémantique.

\subsection{Les unités lexicales à valeur négative ou dépréciative}

\subsubsection{Les déterminants adjectivaux et adverbiaux dépréciatifs}

Un des moyens utilisés pour apporter la contradiction est de rabaisser l'adversaire en utilisant à l'encontre de son travail des termes sémantiquement dépréciatifs tels que : poorly, mistakenly, biased, emotive, confusing, too simplistic, old and outmoded, artificial.

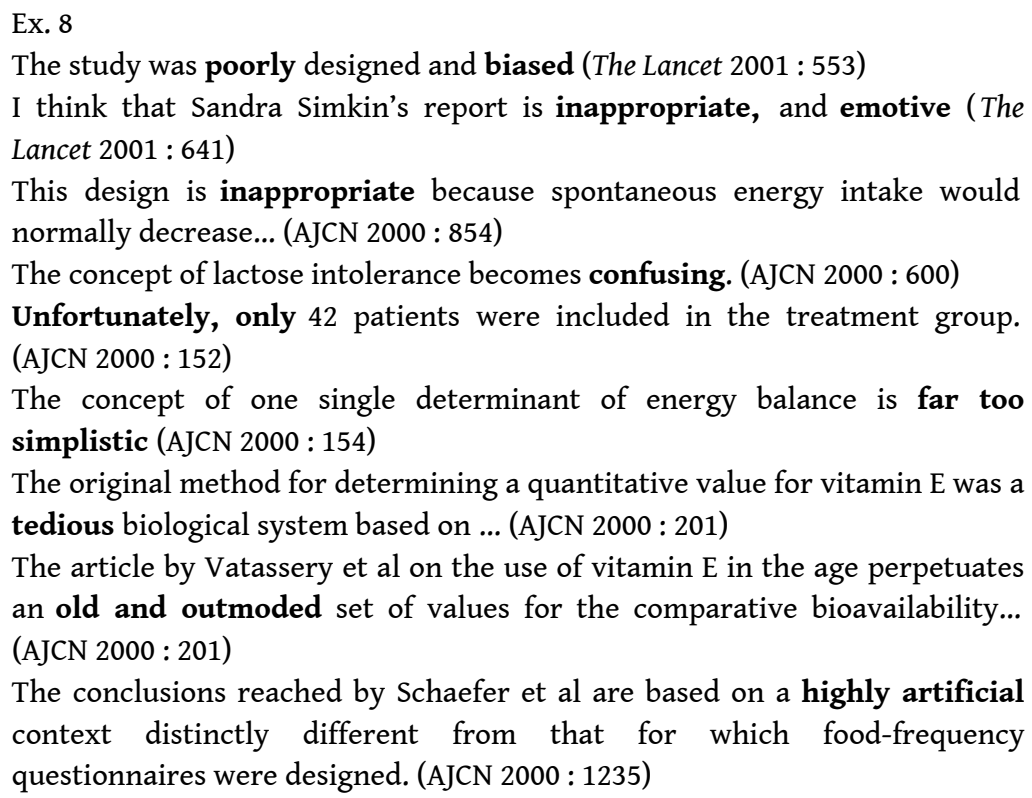

\subsubsection{Les noms et les verbes dépréciatifs}

Notre corpus dénote une utilisation massive de noms et de verbes totalement absents de l'article de recherche, puisque ces termes dépréciatifs sont traditionnellement ceux de la langue des critiques qui portent une forte marque de subjectivité : a critique, a rebuttal, borderline, reductionism, blurring, to refute, to rebut, to fail ...

Ex. 9

The editorial written about our paper by Willett deserves rebuttal because it was essentially a critique of our article. (AJCN 1999 : 572)

In cases in which values are borderline, a repeat test should be conducted. (AJCN $2000: 601$ )

We went on to refute each of Willett's examples with examples that we felt were more appropriate. (AJCN 1999 : 572) 
The sort of reductionism embodied in the interpretation of data from this cohort runs the risk of severely misleading discourse on meaningful public health programs. (AJCN $2000: 850$ )

Thus it is not surprising that there would be a blurring of dietary intakes assessed by ... (AJCN $2000: 1235$ )

\subsection{L'affixation : les préfixes négativants}

Les déterminants adjectivaux sont généralement utilisés dans l'article de recherche pour affiner la notion et ils sont relativement moins nombreux dans le discours scientifique que dans la langue générale. Dans notre corpus, et dans l'état actuel de notre recherche, le recours aux déterminants adjectivaux semble massif, et un grand nombre d'entre eux porte un préfixe négativant dont le but est l'affaiblissement des arguments de l'article de recherche visé. Ces préfixes sont:

in-

inappropriate, inaccurate, inconsistent, incomplete ...

Ex. 10

We felt this was an inappropriate way to analyse the data (AJCN 1999 : 572)

Overall, there are inconsistent data regarding the likely estrogenic and antiestrogenic effects of soy on breast tissue. data (AJCN 1999 : 574)

In vitro systems are incomplete and may not permit ... (AJCN $1999: 574$ )

In conclusion, there are insufficient data on which to draw definite conclusions about... data (AJCN 1999 : 574)

We believe it is incorrect to consider high-fat monoene diets the most healthy choice for sedentary people. (AJCN $2000: 854$ )

At this time, we have insufficient information about potential benefits ...We also have incomplete information about the chemical forms found in foods for use as a guideline in...(AJCN $2000: 1211)$

un-

unreliable, unexpected, unsupported, unclear, unaware...

Ex. 11

Most unexpected however was the unsupported conclusion (The Lancet 2001 : 633)

However, it is unclear whether these epidemiological data, which involved primarily Asian women, can be used to assess the effect of ... (AJCN 1999: 574)

It is unfortunate that Kris-Etherton et al did not report anything about these aspects of their experimental diets. ... (AJCN $2000: 854$ )

out-

outmoded

Ex. 12

The recent review article by [...] on the use of vitamin $\mathrm{E}$ in the aged perpetuates an old outmoded set of values for the comparative study. (AJCN 2000 : 201)

under-

underpowered, understated 
Ex. 13

I believe that their study was underpowered to reach this conclusion (The

Lancet $2001: 553$ )

mis-

misleading

Ex. 14

Cookson's comments and his selective presentation of data are, however, misleading. (The Lancet $2001: 555$ )

The study design used by Schaefer could be highly misleading. (AJCN 2000 : 1235)

\subsection{Les marqueurs exprimant}

\subsubsection{La concession}

Lanalyse linguistique des Letters to the editor permet de relever l'utilisation de marqueurs servant classiquement à l'argumentation, avec en particulier, le recours à des marqueurs d'opposition faible dits concessifs :

although, however, but, yet, nevertheless, nonetheless, even if, even though

Ex. 15

However, they present no sleep records of these patients (The Lancet 2001 :

557)

Although I agree that food-frequency questionnaires are not an optimal method for assessing...the conclusions reached by ... are based on ... (AJCN $2000: 1235)$

\subsubsection{L'antithèse}

L'opposition peut être plus forte et dans ce cas, les marqueurs sont dits d'antithèse : while, whereas, conversely, by contrast, in contrast, otherwise, instead, unlike, opposite

Ex. 16

There was an effect of non-fatal myocardial infarction but not mortality, whereas in GISSI-Prevenzione trial, their opposite seems to have been shown. (The Lancet 2001 : 631)

\subsubsection{La reformulation}

Certains marqueurs servent à la reformulation et s'inscrivent dans la modalité de type 3 (c'est-à-dire de type appréciatif) qui invite au minimum à la réflexion, voire au changement de méthode d'étude. Il s'agit d'une critique polie, voilée...

rather, better, more accurately, in other words

Ex. 17

This finding does not show no risk of DVT for air travel, rather that the risk must be small. (The Lancet $2001: 555$ ) 


\subsubsection{Le doute}

Enfin, la forme la plus faible de la remise en cause explicite est d'émettre des doutes à propos du fait contesté à l'aide de :

maybe, perhaps, probably, highly unlikely, wonder whether

Ex. 18

Perhaps a more pertinent study would be one that investigates why editors feel compelled to challenge not only the management of obesity but the very existence of the disease itself. (AJCN $2000: 845$ )

\section{Les modes de la contradiction implicite} plus subtile et moins directe. Il est toutefois surprenant de noter que ce recours est plus fréquent dans la revue américaine que dans la revue britannique. Ces formes implicites de la contestation seraient-elles à associer à l'une des manifestations du "politiquement correct » pratiqué aux États-Unis, ou faut-il rechercher une explication historique à la différence d'intensité entre les marques de la remise en cause telles que l'on peut les observer dans la revue américaine par rapport à la revue publiée au Royaume-Uni ? Dans tous les cas, cette forme « douce » de la contestation revient à formuler le désaccord de l'énonciateur par des formes modales telles que :

\begin{tabular}{|l|l|}
\hline We find it surprising that ... & \\
Therefore we strongly suggest ... & $\Rightarrow$ I / We disagree \\
Therefore we think... & \\
I have several comments... & \\
\hline I showed clearly... & $\Rightarrow$ I / We are of a different opinion \\
In my opinion... & \\
We believe... & \\
We are aware... & \\
We advocate doing this... & \\
\hline
\end{tabular}

41 L'assertion concernant ses propres résultats avec une forte prise en charge de l'énoncé revient à contredire les résultats de l'étude dont on parle.

\section{Conclusion}

L'expression linguistique des Letters to the editor se démarque fortement de celle de l'article de recherche qui tend à donner une image policée ou polie à son argumentation. $\mathrm{Au}$ contraire, les Letters to the Editor représentent une forme polémique de discours qui témoigne bien de la tension et de la compétition entre les différentes équipes de recherche.

Il est intéressant de noter le caractère beaucoup plus polémique des Letters to the Editor de la revue britannique exprimé en particulier par un recours plus soutenu à des marqueurs linguistiques dépréciatifs. Il serait intéressant de vérifier s'il s'agit là d'une constante de l'expression britannique par rapport à l'usage américain ou s'il s'agit tout simplement d'une spécificité de la revue. Nous sommes actuellement en train d'élargir notre corpus à d'autres revues scientifiques. 


\section{BIBLIOGRAPHIE}

Carston, R. 1998a. « Negation, 'presupposition' and the semantics-pragmatics distinction ». Journal of Linguistics 34, 309-350.

Carston, R. 1998b. « Pragmatics and the explicit-implicit distinction ». Ph.D. Dissertation, University of London.

Cherchi, L. 1983. «Sur la valeur explicative de la notion d'engagement ». Modèles linguistiques V/1, 9, 63-80.

Culioli, A. 1971. Rubriques linguistiques de l'Encyclopédie Alpha. Paris : Grange Batelière.

Culioli, A. 1973. «Sur quelques contradictions en linguistique ». Communications 20, 83-91.

Culioli, A. 1988. « La négation : marqueur et opérations ». Travaux du Centre de Recherches sémiologiques 56, Université de Neuchâtel.

Grize, J. B. \& G. Pieraut-Le Bonniec. 1983. La contradiction. Essai sur les opérations de la pensée. Paris : Presses Universitaires de France.

Halliday, M.A.K. \& J.R. Martin. 1993. Writing Science, Literary and Discursive Power. Pittsburg, PA : University of Pittsburg Press.

Horn, L. R. 1989. A Natural History of Negation. Chicago, IL : University of Chicago Press. 
Huckin, T. N. 1987.« Surprise value in scientific discourse », Communication à la C.C.C. Convention, Atlanta, GA, mars.

Jackendoff, R. S. 1969.« An interpretive theory of negation ». Foundations of Language 5, 218-241

Klima, E. S. 1964. « Negation in English ». In Fodor, J.A. \& J.J. Fatz. (dir.), The Structure of Language. Readings in the Philosophy of Language. Englewood Cliffs, NJ : Prentice Hall Inc, 246-323.

Linebarger, M. C. 1981. The Grammar of Negative Polarity. PhD Dissertation, M.I.D., distributed by Indiana University Linguistic Club.

Lukasiewicz, J. 2000. Du principe de contradiction chez Aristote. Paris : Éditions de l'Éclat.

Magnet, A. 1993. « La discussion de l'article scientifique : quelques aspects linguistiques et discursifs dans trente-six articles de nutrition ». Mémoire de DEA, Bordeaux.

Martin, J. 1996. « Les enjeux du discours scientifique : la stratégie de véridiction ». ASp 11-14, 13-31.

Myers, G. A. 1989. « The Pragmatics of politeness in scientific articles ». Applied Linguistics 10, 1-35.

Myers, G. A. 1990. Writing Biology. Texts as the Social Construction of Scientific knowledge. Madison, WI : University of Wisconsin Press.

Quirk, R., S. Greenbaum, G. Leech \& J. Svartvik. 1985. A Comprehensive Grammar of the English Language. Londres : Longman.

Tournier, J. 1985. Introduction descriptive à la lexicogénétique de l'anglais contemporain. Paris-Genève : Champion Slatkine.

Tournier, J. 1991. Structures lexicales de l'anglais : Guide contemporain. Paris : Nathan.

Wunenburger, J. J. 1989. La raison contradictoire. Science et philosophie modernes, la pensée du complexe. Paris : Albin Michel.

\section{RÉSUMÉS}

Cette communication se fixe comme objectif de repérer quelques stratégies discursives de deux communautés de chercheurs et de comparer leur matérialisation linguistique dans un corpus de Letters to the Editor composé à partir de deux revues : The American Journal of Clinical Nutrition et de The Lancet. La stratégie de remise en cause est analysée comme le mode explicite ou implicite de la contradiction apportée à un fait scientifique établi. Ce discours, identifié comme un genre, se situe dans la logique de véridiction du discours scientifique.

The aim of this study is to pick out a few discursive strategies in two research communities and to perform a comparative analysis of their linguistic content in a corpus of Letters to the Editor selected from two scientific journals: The American Journal of Clinical Nutrition and The Lancet. The strategy of questioning is analysed as an explicit and implicit mode of criticism levelled at an established scientific fact. This discourse, labelled as a genre, is situated in the logic of the veracity of scientific discourse 


\section{INDEX}

Keywords : biology, discourse analysis, letter to the editor, medicine, questioning, veracity Mots-clés : analyse du discours, biologie, contradiction, lettre à l'éditeur, médecine, remise en cause, véridiction

\section{AUTEURS}

\section{ANNE MAGNET}

Anne Magnet est maître de conférences à l'Université de Bourgogne où elle enseigne l'anglais de spécialité à l'U.F.R. des Sciences de la vie. Son domaine de recherche est la linguistique de corpus appliquée aux langues dans le domaine de la biologie. Sa thèse a pour titre : «Étude diachronique de l'article scientifique de recherche scientifique : le cas de la nutrition. Analyse discursive de la construction de l'article. Analyse linguistique des phénomènes de cohésion textuelle ». anne.magnet@u-bourgogne.fr

\section{DIDIER CARNET}

Didier Carnet, agrégé d'anglais, est maître de conférences à la Faculté de Médecine de Dijon. Sa recherche porte sur la linguistique et la pédagogie en anglais médical (enseignement initial et formation continue). Il est le coordinateur de l'ouvrage L'anglais médical à la Faculté (Ellipses 1994), d'un ouvrage thématique sur le vocabulaire médical Words, Sciences de la santé (Ellipses, 2001), ainsi que d'un manuel d'exercices L'anglais pour la santé : Exercices d'appropriation de la langue à visée professionnelle (Ellipses 2001). Il est également l'auteur d'une thèse de doctorat «Opérations de détermination caractéristiques de l'anglais médical » (1996), ainsi que de plusieurs articles sur l'analyse de corpus médicaux. dcarnet@u-bourgogne.fr 